\title{
A Novel Directional Ad Hoc MAC
}

\author{
http://dx.doi.org/10.3991/ijoe.v11i9.5063 \\ Weiquan Wang ${ }^{1,2}$, Zhuxun Song ${ }^{1}$, Xiaofei Mao ${ }^{1}$ \\ ${ }^{1}$ Northwestern Poly Technical University, Xi'an ,China \\ ${ }^{2}$ Sichuan Jiuzhou Electric Group Co., Ltd., Mianyang, Sichuan, China
}

\begin{abstract}
Recently, Ad Hoc network is adapted widely in military, agriculture, and disaster rescue owing to the character flexible and fast deployment without infrastructure itself. However, the omnidirectional Ad Hoc cannot fulfill the requirements from people of increasing the capacity and the bandwidth of network caused by drastic explosion of information. By contrast, the directional antenna is more advantage than the omnidirectional one, which has the capability to improve the performance of Ad Hoc including more transmission range, less interference, spatial reuse, more capacity and tactical silence. Based on the existing lecture, a novel directional MAC found on STDMA ( Spatial Time Division Multiple Access) is raised and provide high throughput, high transmission rate and low delay to network system which contribute to share massive information and improve the performance of the network.
\end{abstract}

Index Terms-Ad Hoc, Directional antenna, MAC, STDMA.

\section{FORWARD}

Ad hoc can construct a temporary selfmanaging system without pre-constructing fixed network infrastructure and consist of a series of wireless terminal which brings flexible deployment and great practicality in some scenario. According to existing study, directional antenna is a wonderful solution deployed in Ad Hoc networks to reduce the signal interference, improve communication quality and performance of the system. A directional Ad Hoc MAC found on STDMA is proposed, and main work includes:

1. Neighbor discovery algorithm under directional antenna model;

2. Directional antenna alignment algorithm;

3. Information sorting algorithm based on priority.

Finally, the MAC is simulated and validated with OPNET which exports the results.

The following article is arranged as follows: section II, describe the detail of MAC, such as the initialization process, neighbor discovery and normal access process. Section III, analyze the main design specifications. Section IV, model the MAC with OPNET. Section V, output the results of analysis and make a conclusion.

In fact, there are so many works focus on directional MAC which is divided into two research directions, the one is to improve the 802.11 protocol family as a supplement, and the other one is to improve ALOHA. In reference [1], D-MAC was proposed by Ko Y and supplemented 802.11 protocol family, in which the RTS was sent by transmitter directionally, and the CTS was Omni-directionally sent from receiver to notice neighbors to block himself in case of collision. Afterward, Roychoudhury R et al raised MMAC protocol, which can afford the communication between nodes far over two hops with multi-RTS, and send CTS/DATA/ACK directionally in one hop. Compare to the D-MAC, MMAC has greater throughput and poorer end to end delay owing to multi-hop communication link. In reference [3], adaptive antenna array was deployed in PHY, and two kinds of control frame were supplemented, in which contains active nodes and topology information about the whole network, respectively. These measures are helpful for nodes to grasp the status of communication nodes and communication link to enhance the RTS addressing probability of multi-hop in MMAC. In reference [4], Takai $M$ et al raised DVCS protocol base on 802.11 DCF, which use directional RTS/CTS to communicate. In reference [5], Lal D et al design a receiver ask mechanism, if some node ready to receive information, it will send the omnidirectional training sequence at first to tell neighbor the size of information can be received itself. A neighbor who received the announcement and have messages to send will send back an RTS packet to a receiver whose size is as the size demanded exactly. After the process, message will be transmitted directionally. In reference [6], the DOA-MAC was raised by Singh $\mathrm{H}$ et al, which is directional MAC based on ALOHA, and divide every slot into three little slot in which node send a tone signal frame, data and confirmation frame. Later, in reference [7] they suggest sending tone signal and receiving tone signal to replace original simple tone signal frame. The mechanism is similar to RTS/CTS handshake protocol, the tone signal from receiver did not indicate the destination node, what is the reason why receiver may receive some data packet not for itself. If the node identity in RTS/CTS can be ignored during handshake protocol, the tone signal frame could be used to displace the RTS/CTS and save control overhead.

\section{DiRECTIONAL MAC}

The MAC protocol takes advantage of STDMA (Spatial Time Division Multiple Access) which ensure the exact communication delay and enhance the reliability of communication. Directional antenna has SDMA character by which system gains the Improvement of tactical communication concealment, spatial reuse and network throughput. Beside, DS and FH are employed in PHY which is good for improving the PLI/PLD of signal further and ensure the communication security.

Design index is as listed:

1. Data rate: $2 \mathrm{Mbps}$ (range: $100 \mathrm{KM}$ ), or $256 \mathrm{Kbps}$ (range: 200KM);

2. End to end sensitive message delay: less than 10ms; 
3. Two level network, maximum capacity: 32 nodes, eight teams in which 2 4 nodes;

4. The communication between Multi-cluster will not be interfered with each other;

5. Access time: less than $5 \mathrm{~s}$;

6. Throughput: over 10Mbps;

7. Radius of network: $200 \mathrm{Km}$.

\section{A. Antenna pattern}

In the system design, the Multi-beam lens antenna is deployed, whose main lobe gain: $21 \mathrm{dBi}$, width: $18^{\circ}$ and pitch angle: $21^{\circ}$. When the antenna works, it switches the beams. To cover three-dimensional space, antenna system consists of three sets of Multi-beam lens antenna which scan together during working and contain 78 beams each, the beam switching time is $10 \mu$ s. Refer to the table 1 which lists the antenna alignment pattern in detail.

TABLE I.

DIRECTIONAL ANTENNA ALIGNMENT MODE

\begin{tabular}{|c|l|l|l|}
\hline $\begin{array}{c}\text { Serial } \\
\text { number }\end{array}$ & Transmitter & \multicolumn{1}{|c|}{ Receiver } & Work scenario \\
\hline 1 & $\begin{array}{l}\text { Directional } \\
\text { transmission }\end{array}$ & $\begin{array}{l}\text { Switch and } \\
\text { simulate } \\
\text { omnidirectional }\end{array}$ & $\begin{array}{l}\text { Network initialization } \\
\text { and sensitive message } \\
\text { transmission }\end{array}$ \\
\hline 2 & $\begin{array}{l}\text { Directional } \\
\text { transmission }\end{array}$ & $\begin{array}{l}\text { Directional } \\
\text { reception }\end{array}$ & $\begin{array}{l}\text { Normal data } \\
\text { transmission slot }\end{array}$ \\
\hline 3 & $\begin{array}{l}\text { Switch and } \\
\text { simulate } \\
\text { omnidirectional } \\
\text { transmission }\end{array}$ & $\begin{array}{l}\text { Switch and } \\
\text { simulate } \\
\text { omnidirectional }\end{array}$ & $\begin{array}{l}\text { Network initialization } \\
\text { and new node join the } \\
\text { network }\end{array}$ \\
\hline
\end{tabular}

The first pattern: under the status that the receiver is not sure the topology information or position of the transmitter, the unify spread code is applied by both sides. If the receiver can't catch the signal by single antenna, the antenna system will turn into joint scan until it does and switch directional receive the pattern.

The pattern is applied during network initialization and sensitive message transmission, to receive information correctly, $\mathrm{K}$ bits leader sequence are added into the frame head to realize antenna alignment, as figure 4 shown. The whole scanning period of systems: $56 * 2 \mathrm{us}=112 \mathrm{us}$, and $\mathrm{K}=112 \mathrm{us} * 2 \mathrm{Mbps}=224$ bits.

The second pattern: the coordinator of system makes an announcement in advance according to the slot schedule and specifies the both sides of every slot before normal communication. When the system works, the transmitter and the receiver will point to each other and communicate.

The third pattern: the both sides of communication will use unify spread code, if the receiver sense the signal, it will switch the beam and aim to the transmitter. The pattern is used mainly in network initialization and new node join network.

In order to simplify the management of the network, the hierarchical structure is employed, which result in two kinds of transmission range: $100 \mathrm{Km}$ and $200 \mathrm{Km}$, the former is applicable to the communication and management in clusters, and the latter suits for communication and management between clusters.

\section{B. Network topology and Information classification}

In the network, hierarchical management is a solution to manage the network structure, which is beneficial to extend network and organize itself. In the structure, network is segmented into clusters which comprise a cluster head and several members, the former can form a higher level network.

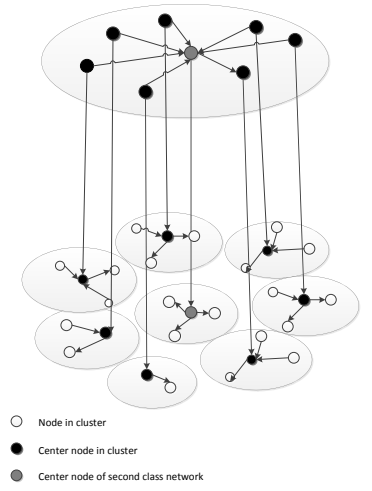

Figure 1. Hierarchical topology structure

In Hierarchical structure, the role of cluster head and member can dynamically exchange. The cluster head is set up to coordinate the communication, route among the cluster, and manage the cluster. Besides, backup cluster head is arranged to enhance the survivability of the network. During running, one normal node can communicate with another one through relaying by cluster head, which facilitate the addressing delay. The Hierarchical structure can be referred in Fig1.

In the team, the temporary center node (cluster head) is selected according to a certain algorithm to coordinate and manage the cluster, distribute slots, release schedule and update topology.

The team temporary center node is selected as the higher level network temporary center node (center head), which is used to coordinate the communication of higher level network, and has the same duty as the temporary center node.

In each level network, the backup temporary center node is selected and activated only if the cluster head is out of work, which strategy is helpful to improve the survivability and the robustness of network. The cluster head and the backup one know well the whole topology and always maintain it.

To ensure real-time communication, and reduce routing time, the rule is defined as follows:

a) In first class cluster, node can communicate with each other straightly;

b) In second class cluster, node can communicate with each other straightly;

c) In a cluster, if communication range is beyond the transmission distance in a cluster, the transmitter can choose a one-hop node which is close to the destination as a relay to realize the communication, or even multihop. If communication is over a cluster, the cluster head, center head and another cluster head who charges the destination could be chosen as relays to realize the transmission. The form of communication includes: unicast (end to end) and broadcast (end to multi-end), the type of information is as shown in table II . 
TABLE II.

INFORMATION TYPE AND SLOT LENGTH

\begin{tabular}{|c|c|c|}
\hline Information type & Length & Slot length \\
\hline $\begin{array}{c}\text { First class time-slot } \\
\text { application data format }\end{array}$ & 327bits & $\begin{array}{l}\text { First class application } \\
\text { time-slot: } 0.6 \mathrm{~ms}\end{array}$ \\
\hline $\begin{array}{l}\text { First class center control } \\
\text { slot data format }\end{array}$ & 1177 bits & $\begin{array}{l}\text { first class center control } \\
\text { slot: } 0.8 \mathrm{~ms}\end{array}$ \\
\hline $\begin{array}{c}\text { C1 network control slot } \\
\text { data format }\end{array}$ & 1501 bits & $\begin{array}{l}\text { C1 network control slot } \\
: 1 \mathrm{~ms}\end{array}$ \\
\hline $\begin{array}{l}\text { Second class time-slot } \\
\text { application data format }\end{array}$ & 775 bits & $\begin{array}{l}\text { Second class } \\
\text { application time-slot: } \\
1.5 \mathrm{~ms}\end{array}$ \\
\hline $\begin{array}{c}\text { Second class center } \\
\text { control slot data format }\end{array}$ & 1669 bits & $\begin{array}{l}\text { Second class center } \\
\text { control slot: } 1.9 \mathrm{~ms}\end{array}$ \\
\hline $\begin{array}{l}\text { Initialization HELLO data } \\
\text { format }\end{array}$ & 183 bits & \\
\hline $\begin{array}{l}\text { Initialization RTT data } \\
\text { format }\end{array}$ & 93 bits & \\
\hline $\begin{array}{l}\text { Access application data } \\
\text { format }\end{array}$ & 97 bits & \\
\hline $\begin{array}{l}\text { Access application } \\
\text { response data format }\end{array}$ & 212 bits & \\
\hline $\begin{array}{c}\text { Access application RTT } \\
\text { data format }\end{array}$ & 93 bits & \\
\hline RTT-REP data format & 213 bits & \\
\hline $\begin{array}{l}\text { Routing control REQ data } \\
\text { format }\end{array}$ & 77 bits & \\
\hline $\begin{array}{l}\text { Routing control REP data } \\
\text { format }\end{array}$ & 77bits & \\
\hline Common data format & $\begin{array}{l}\text { First } \\
\text { class:4402bits } \\
\text {; second } \\
\text { class:5408bits }\end{array}$ & $3.6 \mathrm{~ms}$ \\
\hline $\begin{array}{c}\mathrm{T} \text { time sensitive } \\
\text { information data format }\end{array}$ & 676 bits & $\begin{array}{l}\text { First class: } 0.7 \mathrm{~ms} ; \\
\text { second class: } 2 \mathrm{~ms}\end{array}$ \\
\hline
\end{tabular}

In the protocol, the frame synchronization sequence of data frame is provided by PHY, and the basic size of data frame is 676 bits, if the length of data frame exceeds the basic size, the message will be broken into multiple subframes, and if not, the message will be packaged as basic size.

\section{d) Network initialization}

Before starting, eight nodes are chosen as seeds to trigger the network initialization. First of all, these nodes will keep sending initialization HELLO message, which is designed to discover neighbors. Second, gather all the collected information about neighbors, pick up one as second class temporary center node base on the connectivity algorithm and neighbor information. Third, second class temporary center node collects all the topology information. Fourth, second class center node setup the slot schedule and inform the rest of the node, the second class cluster is built up.

After finishing initializing the second class network, the eight nodes mentioned start to scan the whole spatial space and try to search the remaining node information, include ID, position and so on, after that, build the first class cluster in which the mentioned nodes will be cluster head of first class cluster respectively, the construction procession is similar to the construction of the second class network. The construction of every first class network can be realized at the same time for CDMA, which avoid interfering with each other.

In fact, there is a problem need to be solved in advance that before the network initialization, a start node is appointed, if the node broadcasts the initialization
HELLO, and activate the other seven nodes. The initial procession of second class network will start officially. The detail of initialization is shown as below:

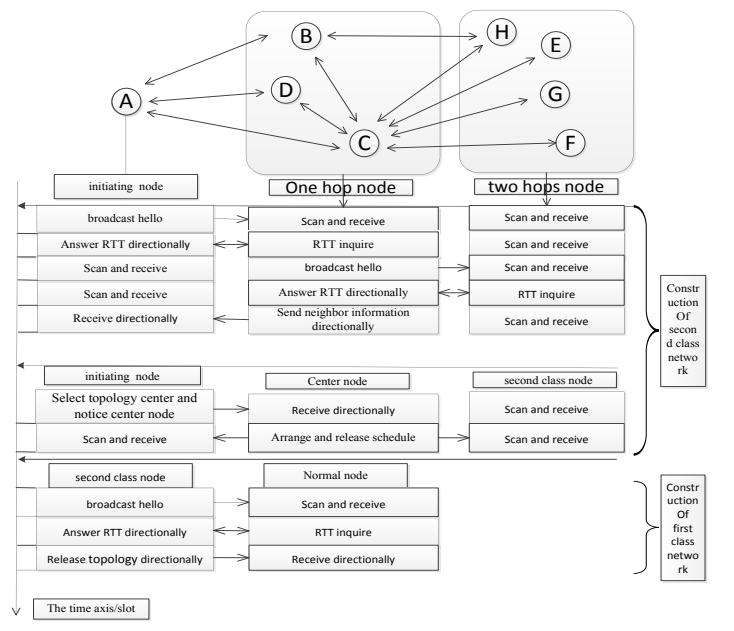

Figure 2. Network initialization

e) Normal access protocol

The normal access slot frame structure is shown in Fig 3. During normal accessing, if the node has a message to send, it will directionally send the message in the specified slot assigned by cluster head, and if not, node switches the antenna function pattern to scan which way is used to listen and receive the time sensitive message in the first class period, time sensitive message and access request message in second class period respectively.

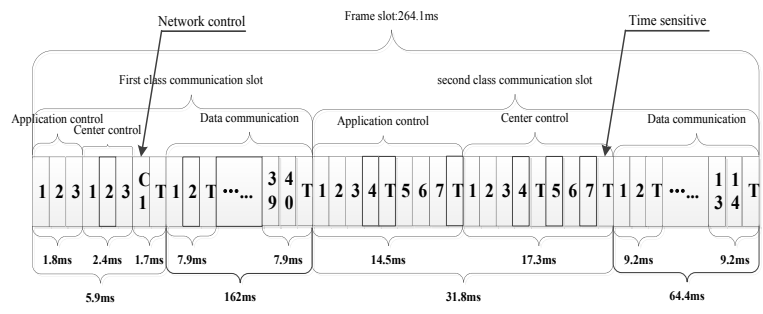

Figure 3. STDMA frame structure

The structure of SDTMA frame includes application control slot, center control slot, network control slot, time sensitive slot and data communication slot.

Application control slot: Set for applying transmission slot and topology updating slot, node apply the slot in the corresponding application control slot, and report its position at the same time.

Center control slot: Set for releasing the slot schedule, access application and network topology. In the slot, cluster head arranges the slot schedule according to business request and issue the schedule, topology information and access application response.

Network control slot: Set for preventing the malfunction or failure of the network center, backup cluster head will take the place of cluster head. In the slot, the backup cluster head backup whole network topology information.

Time sensitive slot: Set for sending time sensitive message.

Data communication slot: Set for three kinds of business data, time sensitive data only take the specialized time sensitive slot. 
The above time slot contains data processing delay and data transmission delay.

To avoid communication interference, CDMA is employed in the MAC protocol. In general, there are two types of spread code, one for network control and access application, and the other for normal communication, by which receiver can tell and process the message with characteristic spread code rapidly.

f) Slot distribution algorithm

In the MAC, slot distribution algorithm bases on the links status, a node applies transmission slot for the priority information in each link concerns itself. There are four types of message in which the first priority is a time sensitive message with exclusive slot. Slot distribution algorithm is arranged for the second to four priority information. The application frame is shown in Fig 4.

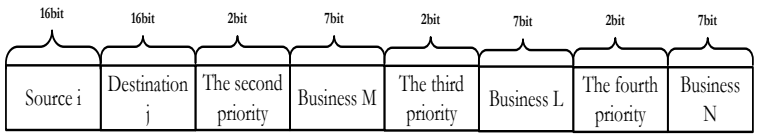

Figure 4. The structure of application frame

The application frame includes source and destination segment, which represents a communication link, and slot number required by each priority transmission, in which M, N, L represents the slot number for each priority respectively. There are four nodes in first class cluster at most which decides maximum of three adjacent links for a node, and there are eight nodes in the second class cluster at most which decides maximum of seven adjacent links for a node in higher level. In the system, the communication way contains unicast and broadcast. According to the maximum information principle, the distribution strategy is broadcast takes priority allocation, after that, the rest of slot is for the same priority information which can be transmitted in parallel. If the slot is not assigned yet, it will be for unicast. The second constraint is high priority information take priority transmission chance, then second priority and lowest priority. If a broadcast is too long to be finished in a remaining slot, it will be postponed for the next slot, the flow chart is as follows.

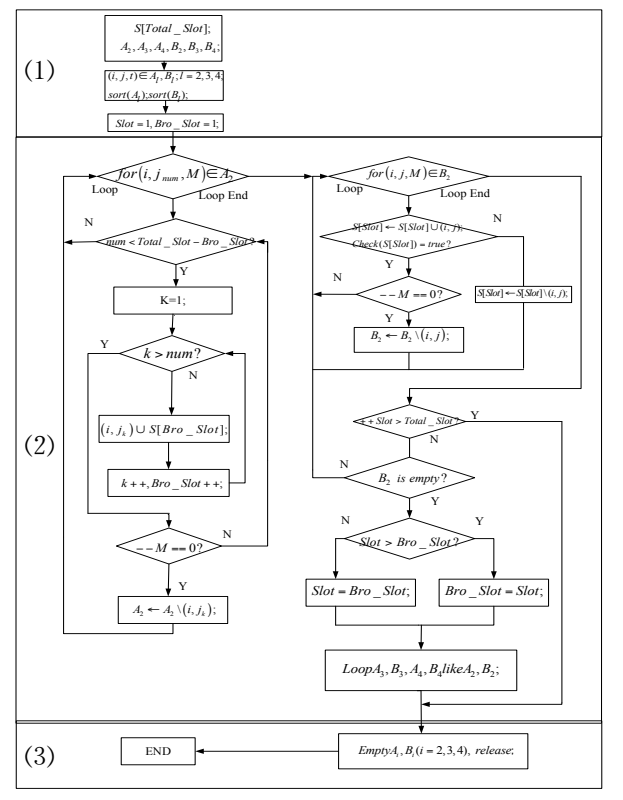

Figure 5. The flow chart of slot distribution
Link set $\left(i, j_{\text {num }}, \mathrm{M}\right)$ represents the link includes broadcast source $i$ and broadcast destination $j_{\text {num }}$, which could be reached in one-hop, $M$ represents the required number of time slots for broadcast. There are six tables, in which A2, A3 andA4 represent the set in different priority with broadcast source $i$ respectively, destination $j_{k}$ and required slot quantity. Similarly, B2, B3 and B4 represent the set in different priority for unicast respectively.

Algorithm description

First: initialization;

Second: allocate slot schedule circularly from $\mathrm{Ai}$ to $\mathrm{Bi}$ until $\mathrm{Bi}$ is empty or a frame slot Total_slot is arranged entirely;

Final: clean out the $\mathrm{Ai}$ and $\mathrm{Bi}$, and release the $\mathrm{S}$ [Total_Slot] schedule.

The first part of the flowchart is initialization process, in which slot and Bro slot are initialized and point to the first slot. Bro_slot always point to the next slot assigned for broadcast, and slot point to the next slot assigned for unicast.

The second part is the core part of the distribution algorithm. In slot distribution, broadcast is given priority to allocate slots first, if there are three links round the broadcast source, the source node have to apply slot for three links once. Poll the table Ai, allocates a slot for the corresponding priority broadcast service and poll the $\mathrm{Bi}$ for unicast as well to gain the maximum parallelism links. After finishing the same priority $\mathrm{Ai}$ and $\mathrm{Bi}$, the slot and Bro_slot will point to next slot for secondary priority $\mathrm{Ai}$ and $\mathrm{Bi}$. In the meantime, if frame slot is over, end the algorithm and empty $\mathrm{Ai}$ and $\mathrm{Bi}$, the message remained will apply for slots in the next round. If the entire table is implemented and table $\mathrm{Bi}(\mathrm{i}=2,3,4)$ is empty, the algorithm will be over either. If the rest of frame slot less than slot quantity, the table Ai may be not empty, which decides the table $\mathrm{Ai}$ is empty or not will not be considered as an influencing factor to end the algorithm.

\section{Performance estimation}

\section{A. Transmission rate}

In a first class network, the end to end range less than $100 \mathrm{Km}$, and the data rate is $4 \mathrm{Mbps}$, in the second class network, the end to end range less than $200 \mathrm{Km}$, and data rate is $2 \mathrm{Mbps}$.

In the normal communication stage, there are 40 data slots which period is $3.6 \mathrm{~ms}$ consist of data transfer time: $3.3 \mathrm{~ms}$, processing and propagation delay: $0.3 \mathrm{~ms}$. Hence, the amount of transmitted information is $3.3 \mathrm{~ms} * 4 \mathrm{M}=13200 \mathrm{bits}$, the period of whole frame is $264.1 \mathrm{~ms}$, the user rate is $13206 * 40 / 0.2641=2.00 \mathrm{Mbps}$. The utilization rate of first class network is $(7.2-0.3 * 2)$ $* 20 / 264.1 \approx 50 \%$ 。

Similarly, there are 14 data slots in which the amount of transmitted information is $676 * 8=5408$ bits, the user rate should be $5408 * 14 / 0.2641 \approx 286.7 \mathrm{Kbps}$. The period of data transmission time slot in the second class network is $3.6 \mathrm{~ms}$ which consist of data transfer time: $2.8 \mathrm{~ms}$, processing and propagation delay: $0.8 \mathrm{~ms}$. The utilization rate of the second class network is $2.8 * 14 / 264.1=14.8 \%$ 。

The total utilization rate of network is $66.8 \%$. 


\section{B. Network throughput}

There are 8 clusters in system network, assume one transfer links at least in the first class communication period and 2 links in second class, time sensitive slot is excluded. The network throughput is

$Q=\frac{13206 \mathrm{bits} * 40 * 8+5408 \mathrm{bits} * 14 * 2}{0.2641 \mathrm{~s}}=\frac{4377344 \mathrm{bits}}{0.2641 \mathrm{~s}}=16.57 \mathrm{Mbps} \approx 17 \mathrm{Mbps}$

\section{PERFORMANCE VERIFICATION}

The MAC is validated with OPNET in which the scene design parameters are as follows:

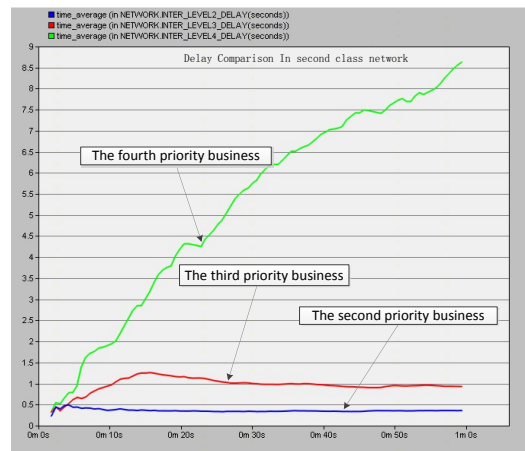

The radius of scene: $250 \mathrm{Km} * 250 \mathrm{Km}$;

The application radius of the network: $200 \mathrm{Km}$;

Data rate: $4 \mathrm{Mbps}$ (first class network): $2 \mathrm{Mbps}$ (second class network);

Node number: 32 nodes, 4 nodes in first class cluster, 8 nodes in second class cluster;

The priority of information classification: four kinds of message, time sensitive message takes the highest priority, voice and data message secondly, and picture message at last;

End to end delay for time sensitive message: less than $8 \mathrm{~ms}$

The output of simulation is as follows:

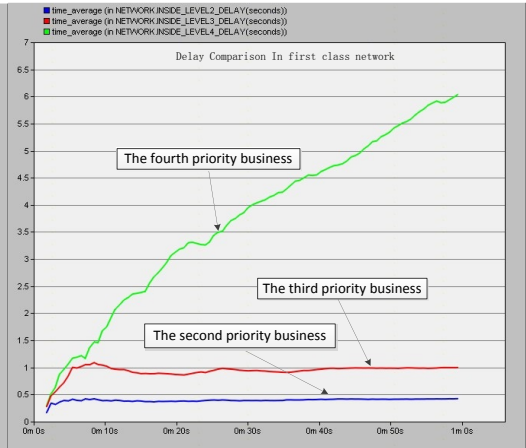

Figure 6. Delay Comparison for all kinds of priority

In Figure 6, the statistical results show the delay not for time sensitive information but latter three kinds of information. The message with higher priority can access channel with lower delay which fit the design requirements. Besides, the access delay of the second and the third priority information is very close, and far below the fourth priority caused by huge amount of former two kinds of priority information. To keep the access balance, the second and third priority information have to be cut down relative to the fourth priority which can avoid of unaccessing of low priority business normally.

The Fig 7 shows that the delay of time sensitive message fluctuates drastically, and tends to be stable gradually. The steady delay is $5 \mathrm{~ms}$ which fits the design requirements. The fluctuation in Fig 7 is caused by equal transmission of all priority information, it's called queuing phenomenon.

As shown in Fig 8, the Average delay of all business is $6.4 \mathrm{~s}$ under the status of equal amount of priority information.

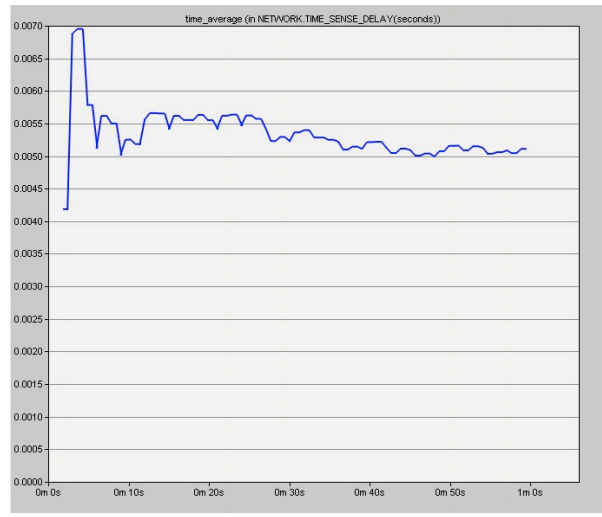

Figure 7. Delay of time sensitive message

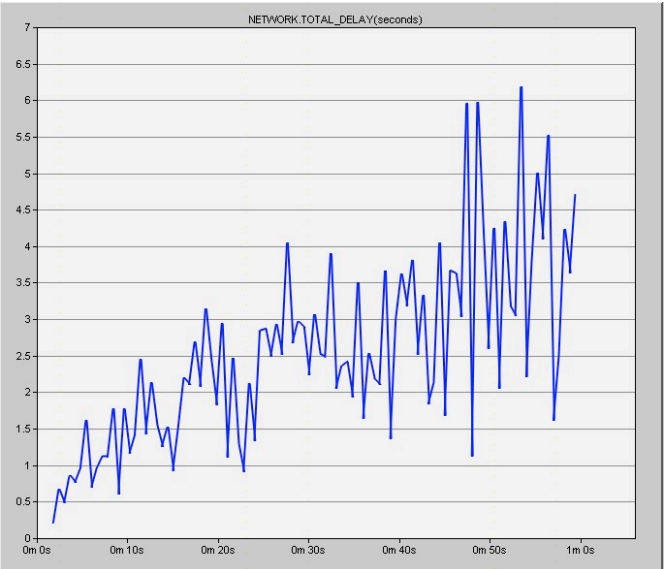

Figure 8. Average delay of all business (time sensitive is excluded)

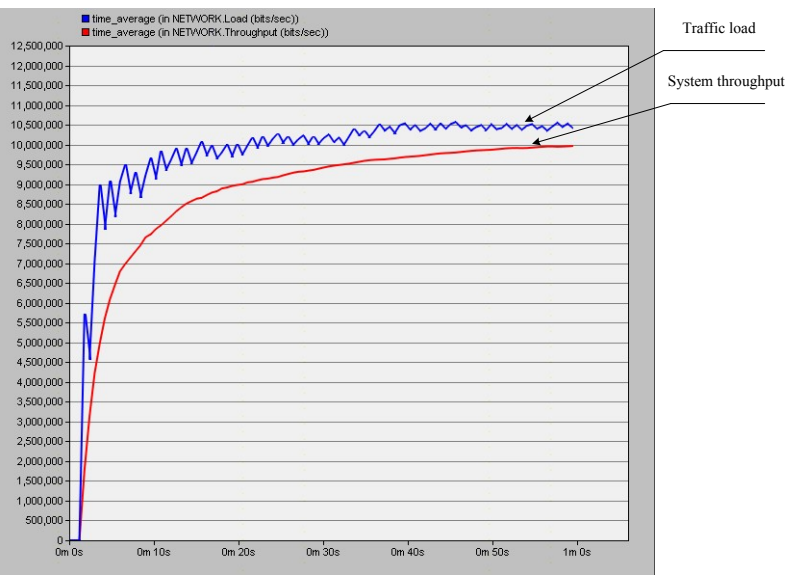

Figure 9. Traffic load and throughput 
From Fig 9, the stable throughput of system is $10 \mathrm{Mbps}$, and the actual traffic load is much higher and reaches $10.5 \mathrm{Mbps}$ which means the performance of system is beyond the original design index.

\section{CONCLUSIONS}

From the above simulation results, the directional MAC proposed can satisfy the system requirements which can ensure time sensitive transmission and improve the throughput with CDMA, SDMA and parallel transmission.

\section{REFERENCES}

[1] Ko Y, Vaidya N H. Medium Access Control Protocols Using Directional Antennas in Ad hoc Networks. Proc. of INFOCOM'00. Tel Aviv, Israel: IEEE Press, 2000: 13-21.

[2] Roychoudhury R, Yang Xue, Vaidya N H, et al. Using Directional Antennas for Medium Access Control in Ad hoc Networks. Proc. of MOBICOM'02. Atlanta, USA: ACM Press, 2002: 59-70. http://dx.doi.org/10.1145/570645.570653

[3] Guama J A, Saad N M. Using Adaptive Antenna Arrays in Mobile Ad hoc Network with Multihop-RTS MAC Protocol. Proc. Of WOCN'07. Singapore: IEEE Press, 2007: 1-4.

[4] Takai M, Martin J, Bagrodia R, et al. Directional Virtual Carrier Sensing for Directional Antennas in Mobile Ad hoc Networks. Proc. of MOBIHOC'02. New York, USA: ACM Press, 2002: 183193. http://dx.doi.org/10.1145/513800.513823

[5] Lal D, Toshniwal R, Radhakrishnan R, et al. A Novel MAC Layer Protocol for Space Division Multiple Access in Wireless Ad hoc Networks. Proc. of ICCCN'02. Miami, USA: IEEE Press, 2002: 614-619. http://dx.doi.org/10.1109/icccn.2002.1043136

[6] Singh H, Singh S. A MAC Protocol Based on Adaptive Beamforming for Ad hoc Networks [C]//Proc. of PIMRC'03. Beijing, China: IEEE Press, 2003.

[7] Singh H, Singh S. Tone Based MAC Protocol for Use with Adaptive Array Antennas. Proc. of WCNC'04. Atlanta, USA: IEEE Press, 2004: 1246-1251. http://dx.doi.org/10.1109/ wcnc.2004.1311367

[8] Fahmy N S, Todd T D. A Selective CSMA Protocol with Cooperative Nulling for Ad hoc Networks with Smart Antennas. Proc. of WCNC'04. Atlanta, USA: IEEE Press, 2004: 387-392. http://dx.doi.org/10.1109/wcnc.2004.1311576

[9] Nasipuri A, Ye S, Hiromoto R E. A MAC Protocol for Mobile Ad hoc Networks Using Directional Antennas. Proc. of WCNC'00.
Chicago, USA: IEEE Press, 2000: 1214-1219. http://dx.doi.org/10.1109/wcnc.2000.904804

[10] Bao L, Garcia J J. Transmission Scheduling in Ad hoc Networks with Directional Antennas. Proc. of MOBICOM'02. Atlanta, USA: ACM Press, 2002: 23-28. http://dx.doi.org/10.1145/ 570645.570652

[11] Gossain H, Cordeiro C, Joshi T, et al. Cross-layer Directional Antenna MAC Protocol for Wireless Ad hoc Networks. Wireless Communication Mobile Computing, 2006, 6(2): 171-182. http://dx.doi.org/10.1002/wcm.377

[12] Takata M, Nagashima K, Watanabe T. A Dual Access Mode MAC Protocol for Ad Hoc Networks Using Smart Antennas. Proc. of ICC'04. Piscataway, USA: IEEE Press, 2004: 4182-4186. http://dx.doi.org/10.1109/icc.2004.1313336

[13] Chen W T, Ho T W, Chen Y C. An MAC Protocol for Wireless Ad-hoc Networks Using Smart Antennas. Proc. of ICPADS'05. Fukuoka, Japan: IEEE Press, 2005: 446-452.

[14] Spyropoulos T, Raghavendra C S. ADAPT: A Media Access Control Protocol for Mobile Ad hoc Networks Using Adaptive Array Antennas [C]//Proc. of PIMRC'04. Barcelona, Spain: IEEE Press, 2004: 370-374.

[15] Choudhury R R, Vaidya N H. Deafness: A MAC Problem in Ad hoc Networks when Using Directional Antennas. Proc. of ICNP'04. New York, USA: IEEE Press, 2004: 283-292. http://dx.doi.org/10.1109/icnp.2004.1348118

\section{AUTHORS}

Weiquan Wang, male, is with Northwestern Poly Technical University, Xi'an 710072, China and Sichuan Jiuzhou Electric Group Co., Ltd., Mianyang, Sichuan, 621000 , China. He was born in 1977, director designer. The main research directions are the data link and the Beidou navigation(6878400@qq.com).

Zuxun Song, male, is with Northwestern Poly Technical University, Xi'an 710072, China. He was born in 1964, professor. The main research directions are electromagnetic compatibility and electromagnetic simulation(philioleo@gmail.com).

Xiaofei Mao, male, $\mathrm{PhD}$ in Reading. He is with Northwestern Poly Technical University, Xi'an 710072, China. The main research directions are the Beidou navigation and Machine Vision. (1011739363@qq.com).

Submitted 21 September 2015. Published as resubmitted by the authors 20 October 2015. 